\title{
Phase Extraction in Dynamic Speckle Interferometry with Empirical Mode Decomposition and Hilbert Transform
}

\author{
S. Equis and P. Jacquot \\ Nanophotonics and Metrology Laboratory, Swiss Federal Institute of Technology Lausanne, EPFL-STI-NAM, Station II, CH-I0I5 \\ Lausanne, Switzerland
}

\begin{abstract}
In many respects, speckle interferometry (SI) techniques are being considered as mature tools in the experimental mechanics circles. These techniques have enlarged considerably the field of optical metrology, featuring nanometric sensitivities in whole-field measurements of profile, shape and deformation of mechanical rough surfaces. Nonetheless, when we consider classical fringe processing techniques, e.g. phase-shifting methods, the deformation range is intrinsically limited to the correlation volume of the speckle field. In addition, the phase evaluation from such patterns is still computationally intensive, especially in the characterisation of dynamic regimes, for which there is a growing interest in a wide range of research and engineering activities. A promising approach lies in the pixel history analysis. We propose in this paper to implement the empirical mode decomposition (EMD) algorithm in a fast way, to put the pixel signal in an appropriate shape for accurate phase computation with the Hilbert transform.
\end{abstract}

KEY WORDS: dynamic speckle interferometry, empirical mode decomposition, Hilbert transform, phase evaluation.

\section{Introduction}

One of the main advantages of whole-field interferometric techniques - at the same time a major source of difficulty - is that a huge amount of information is usually readily available in each frame of a recorded sequence. Each pixel of the photo-detector array acts a priori like an independent sensor, as if the object surface were covered by, say, one million of point detectors. This is a well-known characteristic not only of interferometric techniques, but also of all fringe-based methods, e.g. Moiré, fringe projection and photo-elasticimetry. Nowadays, there is a growing interest within the experimental mechanics community for the characterisation of dynamic regimes, as opposed to static regimes for which the phase extraction is conducted between two steady states. In such dynamic experiment, the phase is most likely to vary at different rates from one pixel to the next. Hence, the processing task consists in handling in parallel that million of non-stationary signals at a temporal sampling rate given by the frame frequency of the camera. A vast domain of time-varying mechanical phenomena, characterised by large temporal frequency bandwidths, is open to investigations as a full range of high-speed digital cameras are available on the market. The only requisite, common to all processing methods, is to fulfil the Nyquist criterion, namely to manage to have less than half a period of the oscillating pixel signal in response to the displacement field induced by the stress increment between any two consecutive frames. To really take advantage of the large amount of data produced easily in this way, efficient signal processing procedures, whose input is either the $2 \mathrm{D}$ pattern or the $1 \mathrm{D}$ temporal pixel signal, are absolutely mandatory.

The focus here is on SI signals, because they exhibit substantial intensity and phase fluctuations, and thus represent the worst case to be treated. However, the method exposed in this paper is not limited to SI signals analysis only. It is applicable to any nonstationary fluctuating signal as soon as there are several oscillations in the whole data set. This method is thus well adapted to deal with any whole-field technique signals, and also to any punctual transducer signals.

Actually, there are many techniques to process fringe patterns in dynamic situations [1]. They are essentially based on phase-shifting, morphological or mathematical transforms approaches. Among them, mention can be made of the object deformationinduced phase shifting technique [2], the local 
interpolation of the intensity pattern using a polynomial [3], the Fourier transform (FT) method combined with spatial carrier fringes [4] and the spiral phase quadrature transform method [5] (basically, a 2D modified Hilbert transform, HT). The FT technique has been extensively and successfully employed and implemented through different variants $[6,7]$. All the frame-based techniques need a 2D unwrapping step, and the task is not trivial with noisy wrapped phase maps. The temporal processing of SI signals is not only the mean to get rid of the intrinsic limit of correlation, but it reduces also the unwrapping task to 1D, much easier than in 2D. The methods addressed to the $1 \mathrm{D}$ temporal problem solving include techniques based on the Morlet wavelet transform [8], the FT [9] and the HT [10].

This paper introduces the empirical mode decomposition (EMD) [11] method as a new way to preprocess the temporal pixel signals. This method has been successfully used in several domains, dealing with strongly non-stationary signals [11-14], and has been introduced lately in SI $[15,16]$. The main contribution of this work lies in a fast implementation of EMD providing well-conditioned signals for an accurate phase extraction by HT.

The paper is organised as follows: in the next section, the HT-based phase extraction of a real-valued signal will be explained in some details. We will review the requirements to extract a physically meaningful phase. The basic principles of the EMD are exposed in a third section, and its usefulness to put a given non-stationary signal in an appropriate shape for phase extraction will be shown. In a fourth part, a fast implementation is explained and vindicated. Experimental results are presented and discussed in the last section.

\section{Phase Extraction from a Real-Valued Signal: The Analytic Method}

Speckle interferometry signals obey the well-known two-beam interference formula:

$i(x, y, t)=i_{0}(x, y, t)+i_{\mathrm{m}}(x, y, t) \cos (\psi(x, y, t))$,

where $i_{0}$ is the mean intensity, $i_{\mathrm{m}}$ the fringe modulation and $\psi$ the phase. These three quantities are random variables, and temporal SI signals in dynamic regimes are thus likely to be strongly non-stationary. As an example, a genuine temporal SI signal is depicted in Figure 1.

The analytic method is probably the most commonly used technique of phase extraction. The

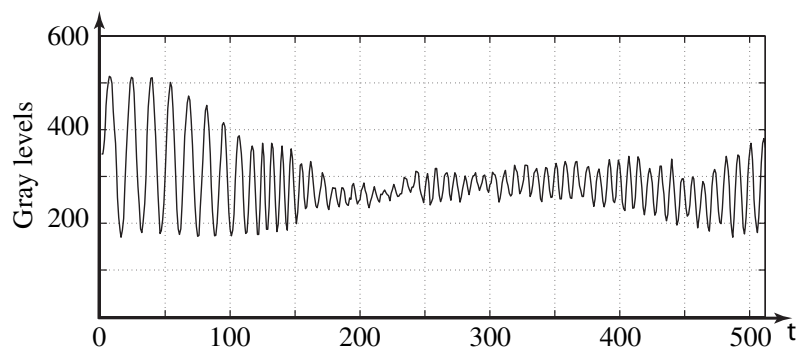

Figure I: A typical temporal pixel signal experimentally obtained in speckle interferometry (grey levels in ordinate and time measured in frame number in abscissa)

analytic signal $z(t)$ is built from the zero mean real valued signal $u(t)$, using the HT:

$\left\{\begin{array}{l}u(t)=\beta(t) \cos (\varphi(t)) \\ z(t)=u(t)-\mathrm{i} \times \mathrm{HT}[u(t)] \\ z(t)=\beta(t) \exp (\mathrm{i} \varphi(t))\end{array}\right.$

As a reminder, the HT of a function $u(t)$ is defined by the following convolution product [17]:

$\mathrm{HT}[u(t)]=\frac{1}{\pi} \int_{-\infty}^{\infty} \frac{u(x)}{t-x} \mathrm{~d} x=\frac{-1}{\pi t} \otimes u(t)$

It is well known that it is much more efficient to compute the convolution product in the Fourier space, thanks to the fast Fourier transform (FFT) algorithm. We thus define the HT in the reciprocal space:

$\operatorname{FT}\{\operatorname{HT}[u(t)]\}=\operatorname{FT}\left\{-\frac{1}{\pi t}\right\} \operatorname{FT}\{u(t)\}=\left\{\begin{array}{ll}\mathrm{i} U(v), & v>0 \\ -\mathrm{i} U(v), & v<0\end{array}\right.$,

where $v$ is the frequency and $U(v)$ is the FT of $u(t)$. Going further into details with the HT is out of the scope of this paper, but it is worth mentioning two basic results for the following:

$\mathrm{HT}[\cos (t)]=-\sin (t)$

$\operatorname{HT}[\alpha]=0, \quad \forall \alpha \in]-\infty, \infty[$

The phase $\varphi(t)$ is then extracted in the range $[-\pi, \pi]$ with the arctan function, from the complex valued signal:

$\varphi_{\mathrm{as}}(t)=\arctan \left(\frac{-\mathrm{HT}[u(t)]}{u(t)}\right)$

There are actually some restrictive conditions in a meaningful use of the HT to get the corresponding analytic signal, or in other words, to extract a phase representative of the physical phenomena: (i) amplitude and phase modulations spectra have to be well separated; (ii) the mean has to be locally zero; and (iii) the signal has to be narrow band. 


\section{Amplitude and phase spectra well separated}

The spectra of the amplitude $\beta(t)$ and of the phase $\varphi(t)$ (Equation 2) have to be well separated (amplitude modulation restricted to low frequencies range, and phase modulation to high frequencies range), otherwise, the computed phase would depend on both, losing physical sense. Bedrosian [18] proposed a product theorem for the HT for band-limited functions. Writing this theorem with the signal $u(t)$ defined in Equation (2) and with Equation (5) leads to:

$$
\begin{aligned}
\operatorname{HT}[u(t)] & =\operatorname{HT}[\beta(t) \cos (\varphi(t))]=\beta(t) \operatorname{HT}[\cos (\varphi(t))] \\
& =-\beta(t) \sin (\varphi(t))
\end{aligned}
$$

The phase is then easily extracted with Equation (7).

In SI, the spectra separation condition is actually intrinsically fulfilled. As can be shown from Figure 1, the modulation depth variations [the term $i_{\mathrm{m}}$ in Equation (1)] are much slower than the oscillation periods of the phase term. Modulation depth variations are ruled by the statistical spatial properties of the speckle fields and decorrelation effects, while the phase variations depend on the sensitivity of the interferometer. Although they form the subject of rather involved mathematical descriptions, the fluctuations of the modulation have been thoroughly studied [19], leading to a set of quite simple operational rules the principal parameters of the optical system - the aperture of the recording system, the pixel size, the sensitivity of the interferometer, the frame frequency and the rate of phase change should obey. It is just reminded here that an inappropriate choice of this set of parameters would lead to a complete failure of the experiment, irrespective of whether processing method could be adopted. On the contrary, with adequately selected system parameters, pixel signals take ipso facto the form of a random, slowly varying modulation depth, enveloping the rapid oscillations of the cosine term. Figure 1 is an archetype of these signals. In speckle interferometry (SI), succeeding in the measurements, fulfilling the correlation conditions and finding the right system parameters are thus three different and equivalent expressions of the same basic phenomena.

\section{Null local mean}

The analytical method fails in the case of real-valued signals with non-zero mean. Indeed the HT of such a signal is given by:

$\operatorname{HT}[\beta \cos (\varphi(t))+\alpha]=-\beta \sin (\varphi(t))$

The extracted phase is then (see Figure 2 representing $z(t)$ in the complex plane):

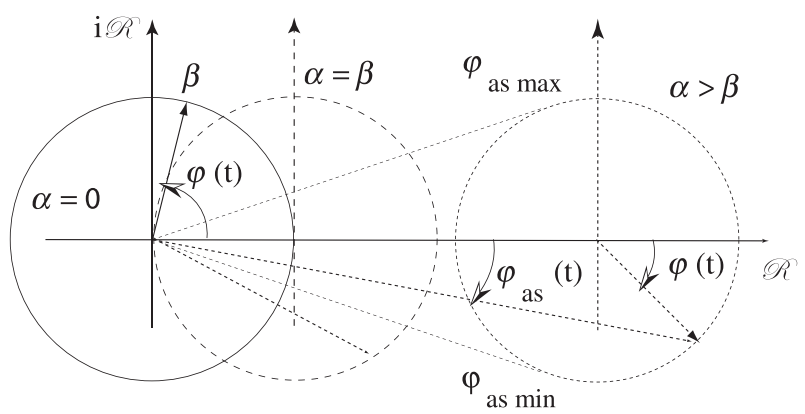

Figure 2: Representation of the analytic signal in the complex plane

$\varphi_{\mathrm{as}}(t)=\arctan \left(\frac{\beta \sin (\varphi(t))}{\beta \cos (\varphi(t))+\alpha}\right) \neq \varphi(t)$

For the dashed curve, the phase moves fast near the origin, with a $\pi$ jump in the limit case where the modulation depth equals the mean, leading to a singularity. As soon as the modulation depth is lower than the mean (dotted line), the phase computed with the HT, $\varphi_{\text {as }}(t)$, is distributed in a narrow range, namely $\left[\varphi_{\text {as min }}, \varphi_{\text {as max }}\right]$, and is indeed very different from the meaningful quantity of interest $\varphi(t)$.

The phase error, when the local mean is not zero but nonetheless smaller than the local modulation depth, can be easily estimated. Let us first rewrite Equation (11) to simplify the notations:

$\varphi_{\mathrm{as}}(t)=\arctan \left(\frac{\sin (\varphi(t))}{\cos (\varphi(t))+\gamma}\right)$,

where $\gamma$, positive and smaller than 1 , represents the ratio mean over modulation. The error between the actual phase $\varphi(t)$, and the unwrapped extracted one $\varphi_{\text {as }}(t)$, is null at $k \pi$ ( $k$ integer), and is thus bounded elsewhere. A Taylor expansion of Equation (11) limited to the first-order readily shows that the error upper bound is equal to $\gamma$ itself. Thus, if we can guarantee a mean envelope, i.e. the mean of the upper and lower envelopes of the signals, much smaller than the modulation depth in the whole data set, the phase error is bounded and does not propagate. In other words, it means that there will be no missed fringe in a real experiment, which is of primary importance.

\section{Narrow-band signal condition}

The HT is perfectly defined for wide-band signals. As soon as its FT exists, there is no difficulty to compute its HT with Equation (4). The problem lies actually in the physical sense to give to the evaluated phase. To illustrate this difficulty, let us consider the two tones signal $u(t)$ with fixed amplitude and constant frequencies: 
$u(t)=A_{1} \cos \left(v_{1} t\right)+A_{2} \cos \left(v_{2} t\right)$,

where $v_{2}=v_{1}+\Delta v$. The two tones case has already been extensively studied, even in the non-stationary situation [20-22]. If $A_{1}=A_{2}$, the phase $\varphi_{\text {as }}$ computed with Equation (7) is found to be the average of $\varphi_{1}=v_{1} t$ and $\varphi_{2}=v_{2} t$. For the unequal strength of the two tones, the phase can even extend beyond the range $\left[\varphi_{1}, \varphi_{2}\right]$. What is true for signals containing two frequencies separated by $\Delta v$ is especially true for signals with a continuous distribution of width $\Delta v$. In fact, this issue is the well-known time-frequency localisation problem. We cannot correctly characterise with one single quantity, the instantaneous phase $\varphi_{\text {as }}$, a signal with a broad frequency content at one time.

In fact, the quantity of interest carried by SI signals is unique at one time as soon as the set-up is mechanically, optically and electronically isolated from external disturbances. Hence, the narrow-band signal condition is fulfilled for the signals we are interested in.

We spent some space to review the requirements that the signal must fulfil to allow a meaningful phase extraction. We focused on the analytical method but the requirements are actually general and apply to any phase extraction or tracking method. We will show now how the EMD method is a perfect candidate to put the signal in the ad hoc shape for subsequent phase evaluation.

\section{Empirical Mode Decomposition: Basic Principles}

\section{Standard algorithm}

Huang et al. [11] proposed in the EMD, which decomposes any non-stationary real-valued signal into its intrinsic oscillation modes, namely the intrinsic modes functions (IMF). The IMFs, that could be non-stationary as well, have to satisfy two conditions: (i) in the whole data set, the number of extrema and the number of zeros differ from each other at most by one; and (ii) the mean envelope is zero.

The first condition is equivalent to the narrowband condition. The second one is a good approximation of the zero local mean condition, and less constraining as it does not need a definition of a local timescale. Indeed, to compute a local mean, we need to define a range containing a sufficient integer number of local periods. For non-stationary signals, the width of this range (local timescale) has to be adjusted all along the time axis. It is directly performed by the EMD algorithm through the extrema finding and the subsequent envelopes computation.
The spectra separation condition for amplitude and phase is fulfilled by the nature of the experiment itself, as previously discussed. Thus, by construction, the IMFs have a well-behaved HT, and more generally they allow good phase extraction.

Basically, the signal is split into a detail part (local higher frequency) and a residue part (local lower frequency):

$s(t)=d(t)+m(t)$,

$d$ and $m$ being, respectively, the local high- and lowfrequency parts. To this aim, the mean has to be estimated through the following procedure.

(1) Identify all extrema of $s(t)$.

(2) Interpolate between maxima (minima) to get an upper (lower) envelope envmax $(t)[\operatorname{envmin}(t)]$ with a certain kernel.

(3) Compute the mean:

$m(t)=[\operatorname{envmax}(t)+\operatorname{envmin}(t)] / 2$

(4) Extract the detail part:

$d(t)=s(t)+m(t)$

Then, the so-obtained component $d(t)$ is the first IMF and the same procedure can be applied to the residue $m(t)$, to extract a second IMF and so on. Actually, the decomposition is not that straightforward and needs an iterative process, namely the sifting process. Let us consider a basic signal composed by a cosine at frequency $\kappa$ and another arbitrary function $\alpha(t)$ which represents the varying bias:

$s(t)=\alpha(t)+\cos (\kappa t)$

The extrema of the raw signal defined in Equation (17) are located at:

$t_{\mathrm{ex}}^{0}=\frac{1}{\kappa} \arcsin \left[\frac{1}{\kappa} \alpha^{\prime}(t)\right]+k \pi \neq k \pi$

where $k$ is an integer and $\beta^{\prime}(t)$ designates the first derivative of $\beta(t)$. At iteration $i$, the extrema locations will change:

$t_{\mathrm{ex}}^{i}=\frac{1}{\kappa} \arcsin \left[\frac{\varepsilon^{i}(t)}{\kappa}\right]+k \pi \neq t_{\mathrm{ex}}^{0}$,

where $\varepsilon^{i}(t)$ is the residual mean envelope at iteration $i$ and should tend to 0 as the number of sifting iterations grows. There is obviously no guarantee that the mean envelope remains the same at any iteration if the extrema locations are different. We have represented in Figure 3, the trend of the ratio upper bound of the mean envelope over modulation depth with respect to the sifting process iteration number, for 


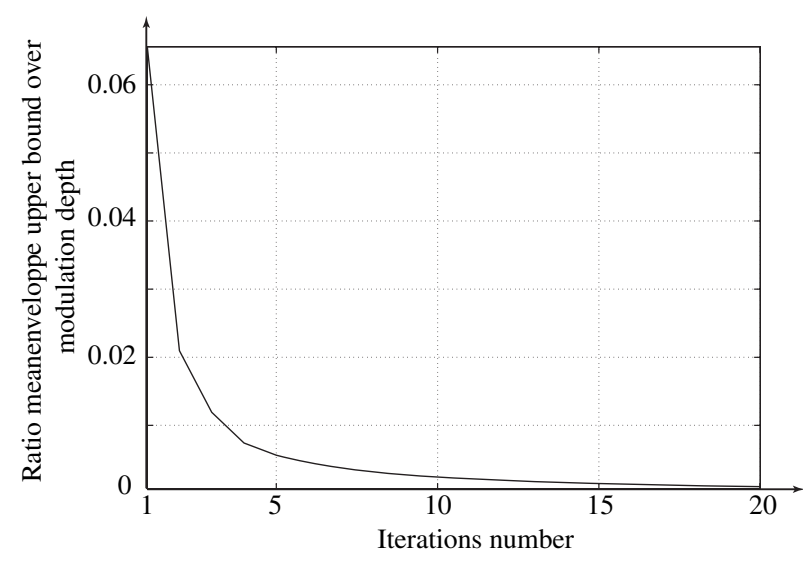

Figure 3: Evolution of the ratio upper bound of the mean envelope over modulation depth with the number of iterations of the sifting process for signal defined in Equation (19)

the following sum of sine waves:

$s(t)=\sin \left(\frac{2 \pi t}{24}\right)+10 \sin \left(\frac{2 \pi t}{1024}\right)$

In this basic example, several iterations are needed to finally get the extrema located at $k \pi$, as it must be for the first sine term which corresponds here to the first IMF. Depending on the sifting process ending criterion, we can readily observe the necessity to proceed with the sifting loop to make the mean envelope below a certain chosen threshold. Thus, this iterative procedure has two purposes: eliminate ridding waves and make the IMF as symmetric as possible (to fulfil the two conditions previously mentioned). The previous procedure $1-4$ is injected into an iterative loop, giving the standard EMD algorithm [12].

(5) If $d(t)$ is an IMF go to 6 (sifting process ending criterion), else go to 1 and proceed with $d(t)$ instead of $s(t)$. As a reminder, $d(t)$ is an IMF if it fulfils the two conditions mentioned at the beginning of this section.

(6) Iterate on the residue $m(t)=s(t)-d(t)$ until the final residue has less than four extrema (at least two extrema are needed for each envelope) or fulfils the EMD ending criterion. We get, at the end, the following final decomposition at the rank $K$ :

$s(t)=\sum_{k=1}^{K} d_{k}(t)+m_{K}(t)$

where the $d_{k}$ are the IMFs and $m_{K}$ is the final residue. For piecewise polynomial signals, the EMD is much sparser than any decomposition on predetermined basis [12]. But, the EMD has the most interesting features when dealing with non-stationary signals. As shown in the example depicted in Figure 4, the parabolic chirp with a varying modulation depth and also a fluctuating bias is well caught by the first IMF. We used here the fast implementation further described in a coming section.
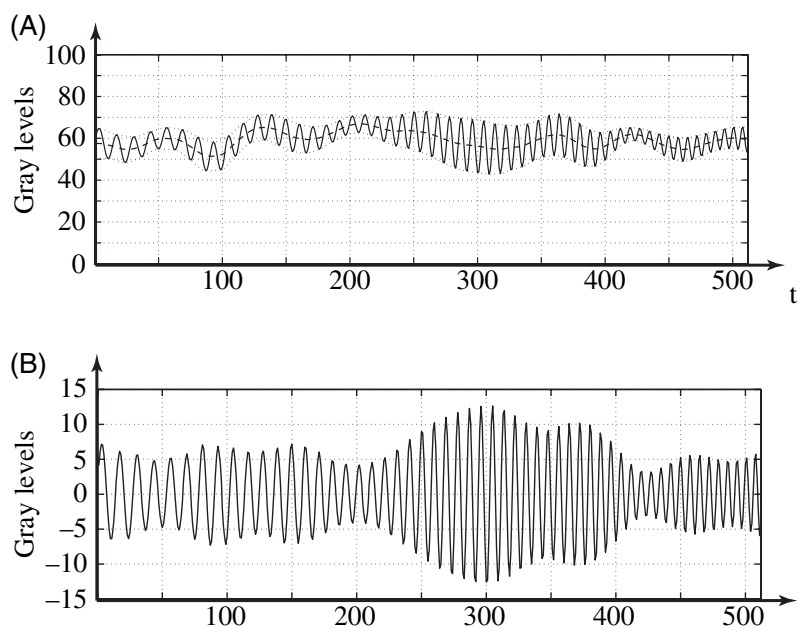

Figure 4: Fluctuating amplitude chirp with varying bias (top) and its first IMF (grey levels in ordinate and time measured in frame number in abscissa)

\section{Issues in the EMD implementation}

One of the main assets of the EMD is its sparseness: the decomposition necessitates indeed much less components to characterise an arbitrary signal than classical Fourier-based or even wavelet-based analysis, especially for non-stationary signals. But one of the main drawbacks lies in the non-uniqueness of the final decomposition. It is indeed strongly dependent on the different algorithm parameters and choices, such as the sifting ending criterion, the boundaries ending technique (signal continuation) and the interpolation method [13].

Indeed, applying the sifting process too many times will over-smooth the mode, resulting in the loss of information and physical meaning. This information will be caught by one or several successive $\operatorname{IMF}(\mathrm{s})$, leading to leakage between modes (information contained in a given frequency band is spread over several modes) and even to overdecomposition. So, a trade-off has to be found, thanks to a judicious sifting stopping criterion.

The boundary ending is also a sensitive part of the mean estimation. An interpolation kernel is chosen to link the extrema with smooth curves. In practice, we used the cubic spline kernel as recommended in Ref. [11]. Some extrapolation is needed near the edges to process the whole data set. To control the envelopes near the boundaries, additional points are thus mandatory at the beginning and end of the data set. In our case, we simply choose to keep at those locations the value of, respectively, the first and last extrema.

In practice, the extrema finding and the interpolation steps on discrete-time signals are quite sensitive, and EMD thus requires a certain amount of oversampling [25]. 
Bad choices for the aforementioned issues could lead to severe errors in the entire decomposition, like overdecomposition but also mode mixing (for instance, higher frequencies oscillations are not caught locally by a given mode but by a successive one that should contain lower frequency oscillations). It is thus mandatory to adapt the algorithm to the type of signals to analyse. Even if the algorithm will lead to IMFs allowing accurate phase extraction from the mathematical point of view, it has to be carefully controlled to give physical meaning to this evaluated phase.

\section{The orthogonality of the decomposition}

At this point, we shall discuss the orthogonality and the completeness of the EMD. Completeness is actually straightforward from the decomposition itself. Orthogonality is a little bit trickier. Let us first rewrite the decomposition in Equation (20) [11]:

$x(t)=\sum_{k=1}^{K+1} C_{k}(t)$

where we consider the last residue as a component. Taking the square of Equation (21) yields:

$x^{2}(t)=\sum_{k=1}^{K+1} C_{k}^{2}(t)+2 \sum_{i}^{K+1} \sum_{j}^{K+1} C_{i}(t) C_{j}(t)$

If the basis vectors form an orthogonal set, the second term of the right member is null. So, we can assess the decomposition orthogonality by computing the following $(K+1) \times(K+1)$ matrices:

$$
\begin{aligned}
\operatorname{OM}(i, j) & =\frac{\sum_{t=0}^{T}\left(C_{i}(t) C_{j}(t)\right)}{\sum_{t=0}^{T} x^{2}(t)} \text { and } \operatorname{OMn}(i, j) \\
& =\frac{2 \sum_{t=0}^{T}\left(C_{i}(t) C_{j}(t)\right)}{\sum_{t=0}^{T}\left(C_{i}(t)^{2}+C_{j}(t)^{2}\right)}
\end{aligned}
$$

where $T$ is the length of the data set. These matrices of orthogonality are symmetric, and even diagonal if the decomposition is orthogonal. On one hand, the matrix OM allows a quick identification of the number of modes of significant energy, and on the other hand, OMn is handy to see qualitatively the leakage between modes.

\section{A Fast and Accurate Implementation of EMD Applied to SI}

For a pixel with enough modulation, the noise remains very low as long as the illumination is well adapted to the sensor dynamic, avoiding thresholding and saturating effects. The relevant information is then carried by the high-frequency part of the signal, i.e. the cosine term in Equation (1), because as already discussed, the fringe modulation, $i_{\mathrm{m}}$ in Equation (1), is always varying much slower than the phase modulation. This information is thus contained in the first IMF, and it becomes useless to proceed further in the modes extraction. We have seen that the sifting process is mandatory to guarantee that the extracted signal is actually an IMF. However, we also briefly showed previously that the phase error is bounded as long as the modulation is greater than the residual local mean value (more precisely the value of the mean envelope). We can then reasonably remove the sifting process to finally extract the first IMF (or pseudo-IMF for the sake of rigorousness) through a single iteration of the EMD algorithm. This strong but meaningful approximation leads to the following algorithm:

1 identify all extrema of the pixel signal $s_{i, j}(t)$, where the couple $(i, j)$ designates a pixel.

2 interpolate between extrema to get the upper $[\operatorname{envmax}(t)]$ and lower $[\operatorname{envmin}(t)]$ envelopes (with the cubic spline kernel).

3 compute the mean:

$$
m_{i, j}(t)=[\operatorname{envmax}(t)+\operatorname{envmin}(t)] / 2,
$$

4 extract the pseudo-IMF:

$$
d_{i, j}(t)=s_{i, j}(t)-m_{i, j}(t)
$$

Thanks to this implementation, we do not have to care about some of implementation issues, such as over-decomposition, leakage, etc. However, it is worth precising that this approximation does make sense as long as some safeguards are put in the algorithm for the extrema finding, interpolation and boundaries ending steps. Phase extraction step is then proceeded on $d_{i, j}(t)$ for each pixel through the use of the HT as detailed previously.

At this point, it is worth mentioning the method developed by Vikhagen [23] and improved by Carlsson and Wei [24] for deformation measurement in dynamic SI experiments. The phase evaluation method [23] consists in scanning the pixel history signal within a local oscillation to detect a maximum value and a minimum value: $\left(i_{0}+i_{\mathrm{m}}\right)$ and $\left(i_{0}-i_{\mathrm{m}}\right)$ with the notations of Equation (1). There is only one unknown left, the phase $\psi$ that is finally easily computed modulo $2 \pi$ at each instant using again Equation (1). A temporal phase shift is added to remove the sign ambiguity of the arcos function. The improvement of the method [24] consists in a better evaluation of the initial speckle phase, i.e. before deformation, and in a least-squares estimation of the 
phase during the deformation allowing at the same time the resolution of the sign ambiguity. Even if Carlsson's method relies on different arguments, it shares with ours the step of extrema finding and mean computation. Yet, the EMD method is likely to be much faster. Indeed, the EMD does not need a lengthy step of signal scanning with a sliding window and the analytic method, although quite sensitive to noise, is also very fast, thanks to the FFT algorithm.

\section{Experimental Results}

To assess the method, we built a classic in-plane SI experiment to measure the deformation of a piece of rubber under compression. The set-up and its geometry are depicted in Figure 5.

The object is illuminated by two divergent laser beams of equal intensity, each of them making an angle $\theta$ with the normal to the object surface. The in-plane deformation is achieved through the use of a PZT actuator, fed with a voltage slope from 0 to $500 \mathrm{~V}$ resulting in a smooth displacement of $40 \mu \mathrm{m}$ of its outer face. For data acquisition, we use a 10-bit CCD camera $1024 \times 1024$ equipped with an objective of $f / 1,4$ as maximum aperture. The camera frame rate is 48 fps. A total of 512 frames are saved on a computer which drives also the PZT actuator. In Figure 5, $S_{1}$ and $S_{2}$ designate the two unit vectors of the illumination directions, while $S_{\mathrm{o}}$ is the unit vector of the observation direction and coincides with the object surface normal. The overall phase change between the two arms when a displacement $\mathbf{L}$ occurs is given by:

$\Delta \varphi=\frac{2 \pi}{\lambda}\left(\vec{S}_{1}-\vec{S}_{2}\right) \vec{L}=S_{x} L_{x}$,

where $S_{x}$ and $L_{x}$ are, respectively, the sensitivity of the interferometer and the projection of the displacement vector on the $x$-axis. Due to its symmetry, the sensitivity is actually simply related to the physical parameters of the set-up by the following relation:

$S_{x}=\frac{4 \pi}{\lambda} \sin \theta$

In our experiment, the sensitivity $S_{x}$ is equal to $9.8 \mathrm{rad} \mu \mathrm{m}^{-1}$. For off-axis points, due to the divergence of the illumination beams, the interferometer has slight sensitivity to $L_{y}$ and $L_{z}$. Those sensitivities are more than one order of magnitude lower than $S_{x}$, and will thus be neglected. The divergent illumination leads also to variations of the sensitivity $S_{x}$ within the field that appear to be negligible (less than $0.2 \%$ ). The mechanical stop can easily be removed in our experiment to produce a pure rigid body motion, what is convenient to conduct a calibration step.

We present in Figure 6 an example of correlation fringes corresponding to the compression. Decorrelation effects are intrinsic to SI and it appears very clearly in this fringe pattern that for displacement even included in the correlation volume, the fringe visibility is very low as soon as the density becomes large (near the mechanical stop in our experiment), preventing any kind of 2D frame-based processing technique. The pixel history based analysis is perfectly able to overcome this limitation.

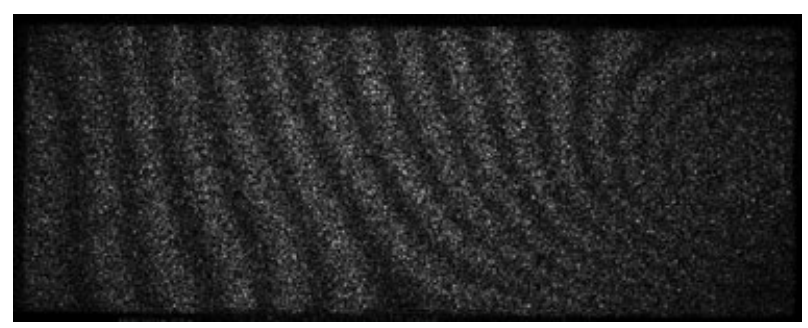

Figure 6: Correlation fringes corresponding to an intermediate state of the $40-\mu \mathrm{m}$ in-plane compression of a piece of rubber
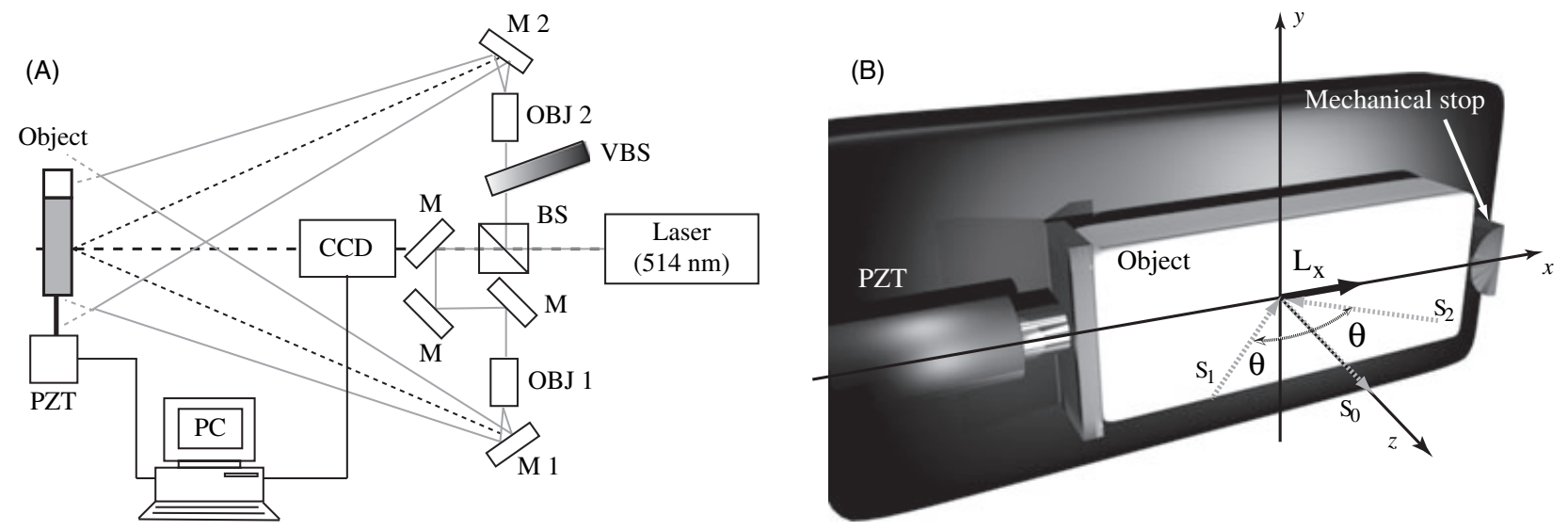

Figure 5: In-plane speckle interferometry experiment 
(A)

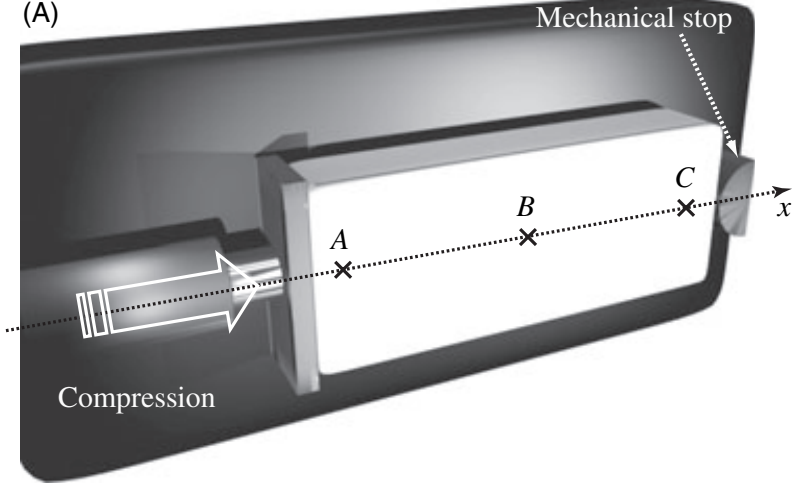

(B)

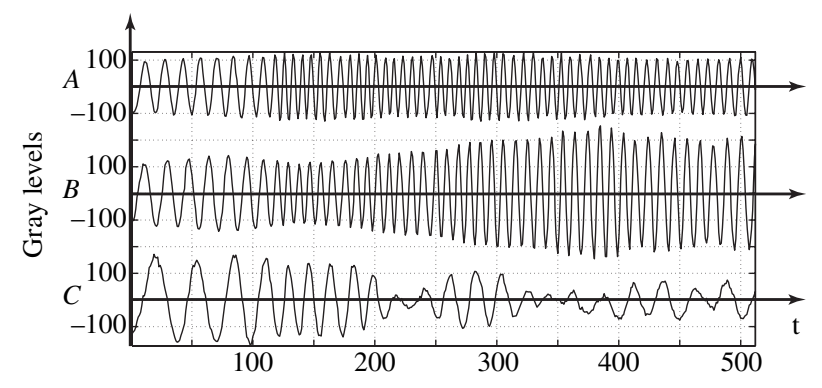

Figure 7: Pseudo-intrinsic modes functions extracted from pixels signals from regions with different displacement velocities (grey levels in ordinate and time measured in frame number in abscissa)

In Figure 7, three-pixel signals processed with our EMD implementation are shown. Those signals come from regions with different displacement velocities: pixel A has the highest number of displacement fringes (60 fringes that is to say a displacement of $38.5 \mu \mathrm{m}), \mathrm{B}$ comes from a lower activity area (47.5 fringes that is to say $30.5 \mu \mathrm{m}$ of displacement), while

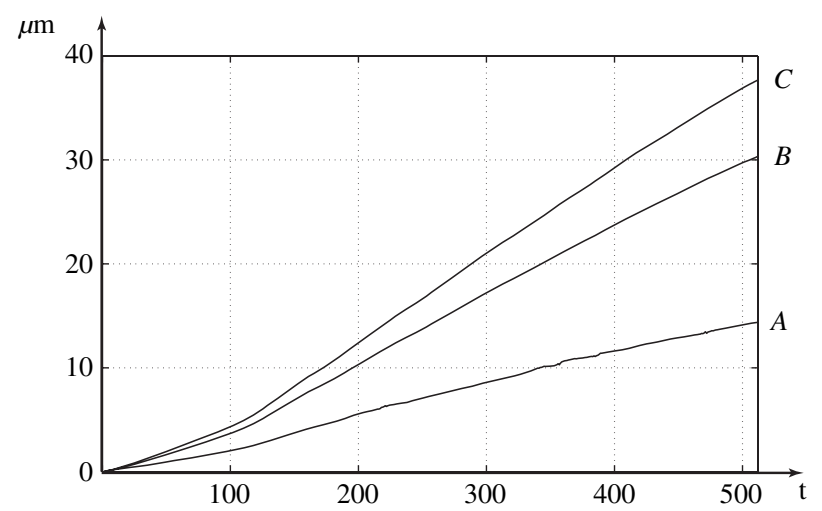

Figure 8: Unwrapped extracted phase for the pixels A, B and $\mathrm{C}(\mu \mathrm{m}$ in ordinate and time measured in frame number in abscissa)

(A)

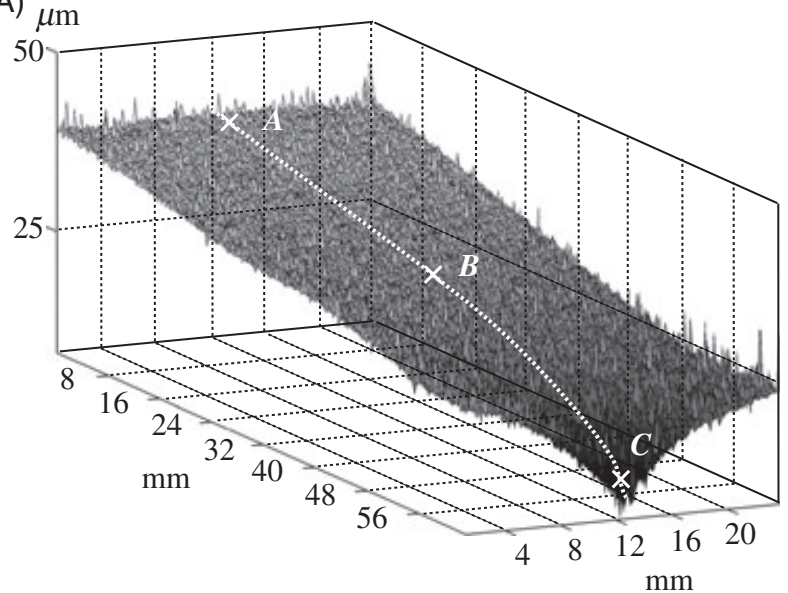

C comes from the mechanical stop neighbourhood (22 fringes that is to say $14 \mu \mathrm{m}$ of displacement). The extracted phase for each pixel A, B and C is depicted in Figure 8.

In Figure 9, the extracted phase of the final state of deformation is depicted and its profile along the $x$-axis in the middle of the object as well. The position of the pixels A, B and C of Figure 7 are also recalled. No temporal carrier has been introduced to conduct this experiment [8], and that is why the noise is quite high around the stop. Pixels signals experience in this area very few fringes of displacement leading to inaccurate phase computation. The method will remain exactly the same if a temporal carrier is added so as to remove the deformation sign ambiguity, and at the same time, to reduce the noise in low activity areas. An additional operation would have to be conducted afterwards to remove the carrier and recover the actual deformation phase, either by calibration or by direct measurement on regions without any activity. There is also some noise due to pixels with low modulation, and we will present in a future work a technique, based on interpolation and

(B)

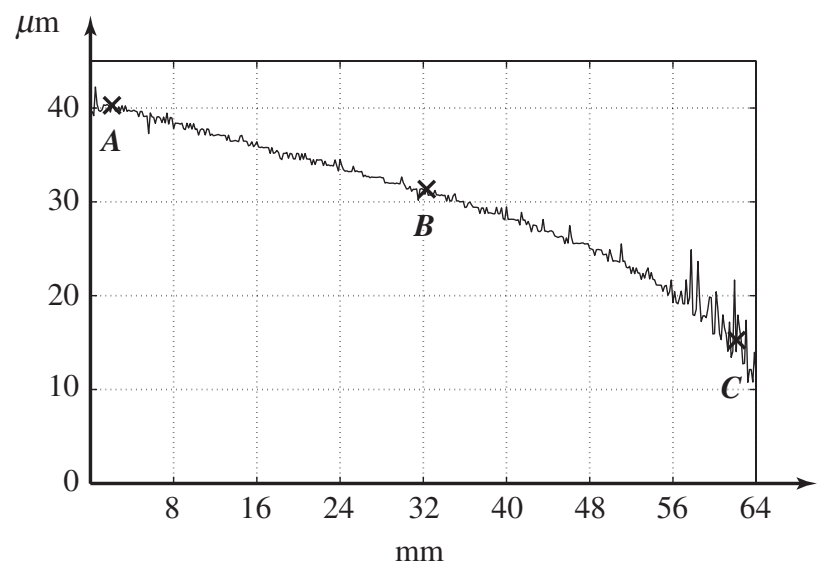

Figure 9: Raw phase of the final state (left) and its profile along the $x$-axis (right) in the middle of the object surface 
non-uniform sampling of the $2 \mathrm{D}$ phase maps, to discard those pixels from which no meaningful information can be extracted.

\section{Conclusion and Outlooks}

This paper introduced the EMD as a new, efficient and flexible processing tool to handle SI and more generally any whole-field technique signals in dynamic regimes. We have shown that this method perfectly matches with the requirements of a meaningful use of the analytic method and more generally of a physically meaningful phase extraction irrespective of the adopted method. The fast implementation without any iterative process has also been vindicated, suggesting possible online implementation for dealing with longer experiments, thereby opening the way for phase extraction and phase tracking methods. Those promising results might allow a more widespread use of SI in the characterisation of dynamic behaviours of mechanical structures.

\section{ACKNOWLEDGEMENT}

This work was supported by the Swiss National Science Foundation.

\section{REFERENCES}

1. Osten, W. and Jüptner, W. (2001) In: Proceeding of the "Fringe" Conferences Series (W. Osten, Ed.). Elsevier, Berlin.

2. Colonna de Lega, X. and Jacquot, P. (1996) Deformation measurement with object-induced dynamic phase-shifting. Appl. Opt. 35, 5115-5121.

3. Robin, E. and Valle, V. (2005) Phase demodulation method from a single fringe pattern based on correlation technique with a polynomial form. Appl. Opt. 34, 7261-7269.

4. Takeda, M., Ina, H. and Kobayashi, S. (1982) Fouriertransform method of fringe-pattern analysis for computerbased topography and interferometry. J. Opt. Soc. Am. 72, $156-160$.

5. Larkin, K. G., Bone, D. J. and Oldfield, M. A. (2001) Natural demodulation of two-dimensional fringe patterns. J. Opt. Soc. Am. A 18, 1862-1881.

6. Bone, D. J., Bachor, H.-A. and Sandeman, R. J. (1986) Fringe-pattern analysis using a 2-D Fourier transform. Appl. Opt. 25, 1653-1660.

7. Baldi, A. and Bertolino, F. (2001) On the Application of the $2 \mathrm{D}$ fast Fourier transform to the surface reconstruction by optical profilometers. XII Int. Conf. Des. Tools Methods Ind. Eng. B1, 9-16.

8. Cherbuliez, M., Jacquot, P. and Colonna de Lega, X. (1999) Wavelet processing of interferometric signals and fringe patterns. Conf. Wavelet Appl. Signal Image Proc. VII. Proc. SPIE 3813, 692-702.
9. Kaufmann, G. H. (2003) Phase measurement in temporal speckle pattern interferometry using the Fourier transform method with and without a temporal carrier. Opt. Comm. 217, 141-149.

10. Madjarova, V. D., Kadono, H. and Toyooka, S. (2003) Dynamic speckle pattern interferometry (DESPI) phase analyses with temporal Hilbert transform. Opt. Exp. 11, 617-623.

11. Huang, N. E., Shen, Z., Long, S. R., Wu, M. C., Shih, H. H., Zheng, Q., Yen, N.-C., Tung, C. C. and Liu, H. H. (1998) The empirical mode decomposition and the Hilbert spectrum for nonlinear and non-stationary time series analysis. Proc. R. Soc. Lond. A 454, 903-995.

12. Rilling, G., Flandrin, P. and Gonçalves, P. (2003) On empirical mode decomposition and its algorithms. IEEEEURASIP Workshop on Nonlinear Signal and Image Processing NSIP-03.

13. Huang, N. E., Wu, M. C., Long, S. R., Shen, S. S. P., Qu, W., Gloersen, P. and Fan, K. L. (2003) A confidence limit for the empirical mode decomposition and Hilbert spectral analysis. Proc. R. Soc. Lond. A 459, 2317-2345.

14. Federico, A. and Kaufmann, G. (2006) Evaluation of dynamic speckle activity using the empirical mode decomposition. Opt. Comm. 267, 287-294.

15. Equis, S., Baldi, A. and Jacquot, P. (2007) Phase extraction in dynamic speckle interferometry by empirical mode decomposition. Proc. Int. Conf. Exp. Mech. ICEM13, 719720.

16. Marengo Rodriguez, F. A., Federico, A. and Kaufmann, G. H. (2007) Phase measurement improvement in temporal speckle pattern interferometry using empirical mode decomposition. Opt. Comm. 275, 38-41.

17. Bracewell, R. (1965) The Fourier Transform and Its Applications. McGraw-Hill Book Company, New York.

18. Bedrosian, E. (1963) A product theorem for Hilbert transforms. Proc. IEEE 51, 868-869.

19. Lehmann, M. (2001) Speckle statistics in the context of digital speckle interferometry. In: Digital Speckle Pattern Interferometry and Related Techniques, Chapter 1 (P. K. Rastogi, Ed.), John Wiley \& Sons, Ltd, Chichester: $1-58$.

20. Mandel, L. (1974) Interpretation of Instantaneous Frequencies. Am. J. Phys. 42, 840-846.

21. Loughlin, P. J. and Tacer, T. (1996) On the amplitude- and frequency-modulation decomposition of signals. J. Acoust. Soc. Am. 100, 1594-1601.

22. Boashash, B. (1992) Estimating and interpreting the instantaneous frequency of a signal. Proc. IEEE 80, 520568.

23. Vikhagen, E. (1990) Nondestructive testing by use of TV holography and deformation phase gradient calculation. Appl. Opt. 29, 137-144.

24. An, W. and Carlsson, T. E. (2003) Speckle interferometry for measurement of continuous deformations. Optics Lasers Eng. 40, 529-541.

25. Rilling, G. and Flandrin, P. (2006) On the influence of sampling on the empirical mode decomposition. IEEE Int. Conf. Acoust. Speech Signal Proc. ICASSP-06, SPTM-P5.11. 